\title{
Aftermath of explosions in underground free space
}

\author{
P. P. Procházka \& A. N. Kravtsov \\ Association of Czech Civil Engineers and Czech Technical University in \\ Prague, Civil Engineering, Structural Mechanics, Czech Republic
}

\begin{abstract}
Today's world has a very urbanized structure - more than half the population of 6,8 billion people live in cities, and underground spaces are one of the most prerogative zones for city's of modern civilization. Underground spaces have grown, along with the risk of terrorism and its organization. Therefore, it is very important to predict the dynamic impacts of explosives in underground spaces and the blast waves can be transformed into a loading of solid structures. In this paper, the impact of explosions and air strike waves is formulated and solved. The location of the center of explosion is very relevant. It appears that if the location is at the solid surface, this part can be up to $30 \%$ of the total energy (in soft solids). The variables to be calculated are the mass density of gas, the velocity of movements and the internal energy. The latter covers the influence of the gas pressure, being given for the adiabatic state. The air is linearly related to the internal energy of a unit mass of the gas, as is the density, while in the neighborhood of the source of explosion, the pressure changes nonlinearly with respect to the gas density.
\end{abstract}

Keywords: underground spaces, strike wave, governing equations.

\section{Introduction}

The objective of this paper is to create an algorithm for calculating the dynamic movement of a strike wave in space and its influence on underground structures. Such a problem is very complicated from the point of view of both theoretical formulation and numerical algorithm development. This is why a simplification is made, but still the result is nonlinear in almost each equation. First the 
constitutive equations of gas dynamics are recorded and then the solution of this problem is suggested.

In order to describe such displacements of the gas, a couple of numerical approaches exist. One of the most suited appears to be the smooth hydrodynamics particle method (SHP), which was historically developed for astrophysical purposes, [1, 2]. The inherent benefit of the SHP formulation consists in the transformation of partial differential equations to a system of linear algebraic using regularization. This transformation is, among others, suitable for parallel computations. Recently, SHP has grown into a successful and respected numerical tool. In particular, this method does not differ between 3D, 2D and 1D problems, as the problems defined in higher order spaces can be simulated as easy as those in 1D. An excellent review of the advantages and recent progress in SHP can be found in [3, 4]. Some problems occur when geometrical boundary conditions should be involved. The authors of [5] proposed the ghost particle method, in which some particles are located outside the domain. The heat conduction problem is solved in [6], where Taylor series expansion approximates the regularization kernels.

This paper partly starts with ideas of Veselovsky et al. [7], where the problem of an explosion in underground parking is solved. More detailed analysis is submitted in [8], where two-dimensional problems of gas dynamics are comprehensively discussed. The general approach for hydrodynamic processes involving strike waves and high temperature can be found in [9]. This information is collected into a formulation of loading, acting against fixed walls of underground parking. The solution of the problem is done in terms of SHP.

\section{Equations of gas dynamics}

Mathematical modeling of the air movements is based on the solution of equations of gas dynamics, which for a three-dimensional problem in a Cartesian system of coordinates are listed as:

$$
\begin{gathered}
\frac{\partial \rho}{\partial t}+\frac{\partial\left(\rho u_{x}\right)}{\partial x}+\frac{\partial\left(\rho u_{y}\right)}{\partial y}+\frac{\partial\left(\rho u_{z}\right)}{\partial z}=\frac{\mathrm{d} \rho}{\mathrm{d} t}+\rho \operatorname{div} U=0 \\
\frac{\partial\left(\rho u_{x}\right)}{\partial t}+\frac{\partial\left(p+\rho u_{x}^{2}\right)}{\partial x}+\frac{\partial\left(\rho u_{x} u_{y}\right)}{\partial y}+\frac{\partial\left(\rho u_{x} u_{z}\right)}{\partial z}=\frac{\mathrm{d}\left(\rho u_{x}\right)}{\mathrm{d} t}+\frac{\partial p}{\partial x}+\rho u_{x} \operatorname{div} U=0 \\
\frac{\partial\left(\rho u_{y}\right)}{\partial t}+\frac{\partial\left(\rho u_{x} u_{y}\right)}{\partial x}+\frac{\partial\left(p+\rho u_{y}^{2}\right)}{\partial y}+\frac{\partial\left(\rho u_{y} u_{z}\right)}{\partial z}=\frac{\mathrm{d}\left(\rho u_{y}\right)}{\mathrm{d} t}+\frac{\partial p}{\partial y}+\rho u_{y} \operatorname{div} U=0 \\
\frac{\partial\left(\rho u_{z}\right)}{\partial t}+\frac{\partial\left(\rho u_{x} u_{z}\right)}{\partial x}+\frac{\partial\left(\rho u_{y} u_{z}\right)}{\partial y}+\frac{\partial\left(p+\rho u_{z}^{2}\right)}{\partial z}=\frac{\mathrm{d}\left(\rho u_{z}\right)}{\mathrm{d} t}+\frac{\partial p}{\partial z}+\rho u_{z} \operatorname{div} U=0 \\
\frac{\partial e}{\partial t}+\frac{\partial\left[(e+p) u_{x}\right]}{\partial x}+\frac{\partial\left[(e+p) u_{y}\right]}{\partial y}+\frac{\partial\left[(e+p) u_{z}\right]}{\partial z}= \\
=\frac{\mathrm{d}(e+p)}{\mathrm{d} t}+(e+p) \operatorname{div} U-\frac{\partial p}{\partial t}=0
\end{gathered}
$$


where (1) describes the continuity equations, (2) are three equations of impulses (listed in 3D) and (3) shows the energy equilibrium. In the above equations the following are denoted:

$x, y, z$

$U \equiv\left\{u_{x}, u_{y}, u_{z}\right\}$

$u_{x} \equiv u_{x}(x, y, z, t)$

$u_{y} \equiv u_{y}(x, y, z, t)$

$u_{z} \equiv u_{z}(x, y, z, t)$

$\rho \equiv \rho(x, y, z, t)$

$p \equiv p(x, y, z, t)$
Cartesian coordinates [m]

vector of velocity $U,[\mathrm{~m} / \mathrm{msec}]$

$e=\rho\left[\varepsilon-\left(u_{x}^{2}+u_{y}^{2}+u_{z}^{2}\right) / 2\right]$ full energy of a unit of mass of the gas, [MPa]

$\varepsilon \equiv \varepsilon(x, y, z, t)$ the potential energy density $\left[\mathrm{m}^{2} / \mathrm{ms}^{2}\right]$

$\frac{1}{2}\left(u_{x}^{2}+u_{y}^{2}+u_{z}^{2}\right)$ the density of gas $\left[\mathrm{kg} / \mathrm{m}^{3}\right]$ the pressure of gas $[\mathrm{MPa}]$ the kinetic energy density $\left[\mathrm{m}^{2} / \mathrm{ms}^{2}\right]$

$\frac{\mathrm{d}}{\mathrm{d} t}=\frac{\partial}{\partial t}+u_{x} \frac{\partial}{\partial x}+u_{y} \frac{\partial}{\partial y}+u_{z} \frac{\partial}{\partial z} \quad$ is the substantial derivative

For ideal gas it approximately holds:

$$
\varepsilon=\frac{p}{\rho(\gamma-1)} \Rightarrow e+p+K=\frac{\gamma}{\gamma-1} p+K
$$

providing the exponent of the adiabatic process $\gamma(\rho)$ is defined as:

$$
\begin{aligned}
& \gamma=3 \quad \text { if } \rho>440 \mathrm{~kg} / \mathrm{m}^{3} \\
& \gamma=1.3 \quad \text { if } \rho<50 \mathrm{~kg} / \mathrm{m}^{3} \\
& \gamma=\gamma_{0}(\rho) \text { if } 50 \leq \rho \leq 440\left[\mathrm{~kg} / \mathrm{m}^{3}\right]
\end{aligned}
$$

where $\gamma_{0}$ is the linear approximant for the given interval, and $K$ is the kinetic energy.

Equations (1)-(3) create a simultaneous system of nonlinear equations. For the next consideration denote $D u=\frac{\mathrm{d} u}{\mathrm{~d} t}$ and $D_{0}=\frac{\partial}{\partial t}+u_{x}^{0} \frac{\partial}{\partial x}+u_{y}^{0} \frac{\partial}{\partial y}+u_{z}^{0} \frac{\partial}{\partial z}$. Then the first equation turns to

$$
D_{0} \rho+\rho_{0} \operatorname{div} U=0
$$


where $\rho_{0}$ is a reference value of the mass density and $\rho$ is in fact a small deviation (fluctuation) of this quantity. The second system may lead to the triple equations:

$$
\begin{aligned}
& D_{0} u_{x}+\frac{1}{\rho_{0}} \frac{\partial p}{\partial x}=0 \\
& D_{0} u_{y}+\frac{1}{\rho_{0}} \frac{\partial p}{\partial y}=0 \\
& D_{0} u_{z}+\frac{1}{\rho_{0}} \frac{\partial p}{\partial z}=0
\end{aligned}
$$

and the divergence obviously disappears. In the last equation the new constant $c_{0}$ rises instead of $\gamma$ :

$$
D_{0} p+\rho_{0} c_{0}^{2} \operatorname{div} U=0
$$

From (4) and (6) it immediately follows that:

$$
D_{0}\left(p-c_{0}^{2} \rho\right)=0
$$

From the latter equation one gets that there is a relation between pressure and density, and consequently, only four unknowns are to be solved, $\left\{u_{\mathrm{x}}, u_{y}, u_{z}\right\}$ and the density (or alternatively pressure).

\section{Methodology of formulation of the loading}

The approach is divided into two steps. In the first stage the initial state of explosion and spread of the air strike wave until the moment of contact with the structures is solved.

The calculation of strike wave parameters at the beginning stage is based on the numerical calculation of one dimension equations of the gas dynamic. The distribution of density $\rho(\boldsymbol{r})$, velocity $\boldsymbol{u}(\boldsymbol{r})$ and internal energy $\varepsilon(\boldsymbol{r})$ ( $\boldsymbol{r}$ is the radius or distance from the origin (charge), which is centered at the point of explosion) at the moment of the beginning of the interaction of the air strike wave with the nearest structures used for the calculation of spread out and various interactions among air strike waves are incorporated in the interfacial conditions with the second stage.

In the second step processes of interaction of the air strike waves with structures can be studies. Strike wave parameters appearing in the second step are computed from equations of the three-dimensional gas dynamic.

The problem is solved by the smooth hydrodynamic particles method; see [10], for example. 


\section{Examples}

A special explosive scheme of the three dimensional problem is considered in this section. It starts with the position of the charge near the neighboring side walls, Fig. 1 . The ball centered at the charge position with radius $R_{\mathrm{c}}$ describes the domains of charge in the picture.

The charge position, as well as the side walls, ground and ceiling, are imbedded in Cartesian coordinates $0 x y z$, where the plane $0 x y$ is the ground and $z$ is upwards oriented. In both cases the ground is characterized by the plane $z=0$ and the ceiling is the plane $z=3$. The length dimensions are measured in meters. The center of the charge possesses the coordinates $\left(0.5+R_{\mathrm{c}}, 0.5+R_{\mathrm{c}}, 0.5+R_{\mathrm{c}}\right)$ in the first case and in the second case the vertical coordinate is $0.5+R_{\mathrm{c}}$, while the other coordinates are zero. Values of the radii of charge and its mass $q$ are introduced in Table 1 for the density of TNT $\rho_{\mathrm{TNT}}=1620 \mathrm{~kg} / \mathrm{m}^{3}$.

The influence of loads is depicted for the air explosion charges of mass $q=50$ $\mathrm{kg}$ and $q=100 \mathrm{~kg}$ TNT is shown in Fig. 2.
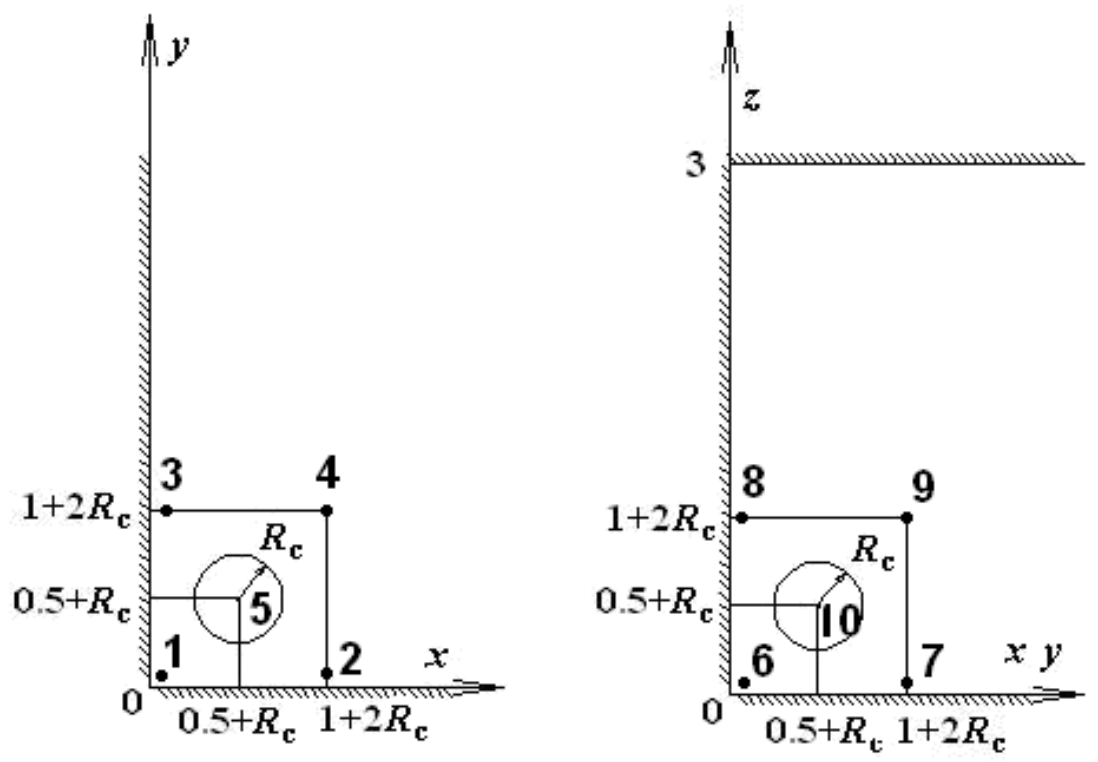

Figure 1: $\quad$ Scheme for the charge position near the side walls.

Table 1: $\quad$ Dependency of the radius $R_{\mathrm{c}}$ on the power $q$.

\begin{tabular}{|c|c|}
\hline$q, \mathrm{~kg}$ & $R_{\mathrm{c}}, \mathrm{m}$ \\
\hline 50 & 0.245 \\
\hline 100 & 0.195 \\
\hline
\end{tabular}



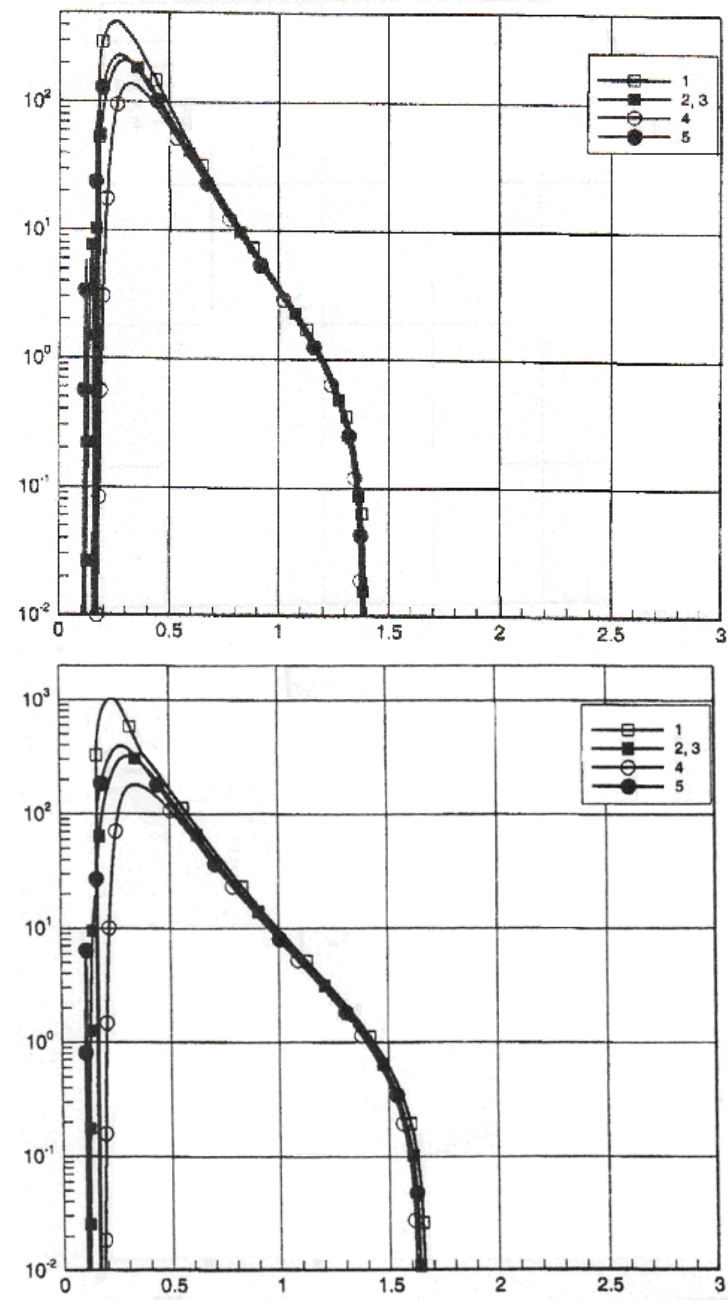

Figure 2: Development of pressure $p\left[\mathrm{~kg} / \mathrm{cm}^{2}\right]$ on the ground in time [msec]. Upper picture: $q=50 \mathrm{~kg}$, lower picture: $q=100 \mathrm{~kg}$.

\section{Conclusions}

The influence of an explosion in an underground spare (say underground parking, subway station, etc.) on the ground and side walls is studied in this paper. In comparison to a previous paper of the authors, here direct effect on the solid parts (being considered as stiff enough) of the underground structure is observed in dependency of time scale. From the numerical computation, based 
on SPH (smooth particle hydrodynamics method) one can see that because of the very rapid velocity of the explosion, a sudden increase of the pressure is obtained in the first second, but the effect decreases almost immediately, here it is about 1.5 until $2 \mathrm{msec}$. From this fact the structures being exposed to possible explosive loading can be designed and assessed and, moreover, in order to ensure a proper dissipation of energy, decelerators can be positioned at the right places and demands on the properties of them can also be fulfilled correctly.
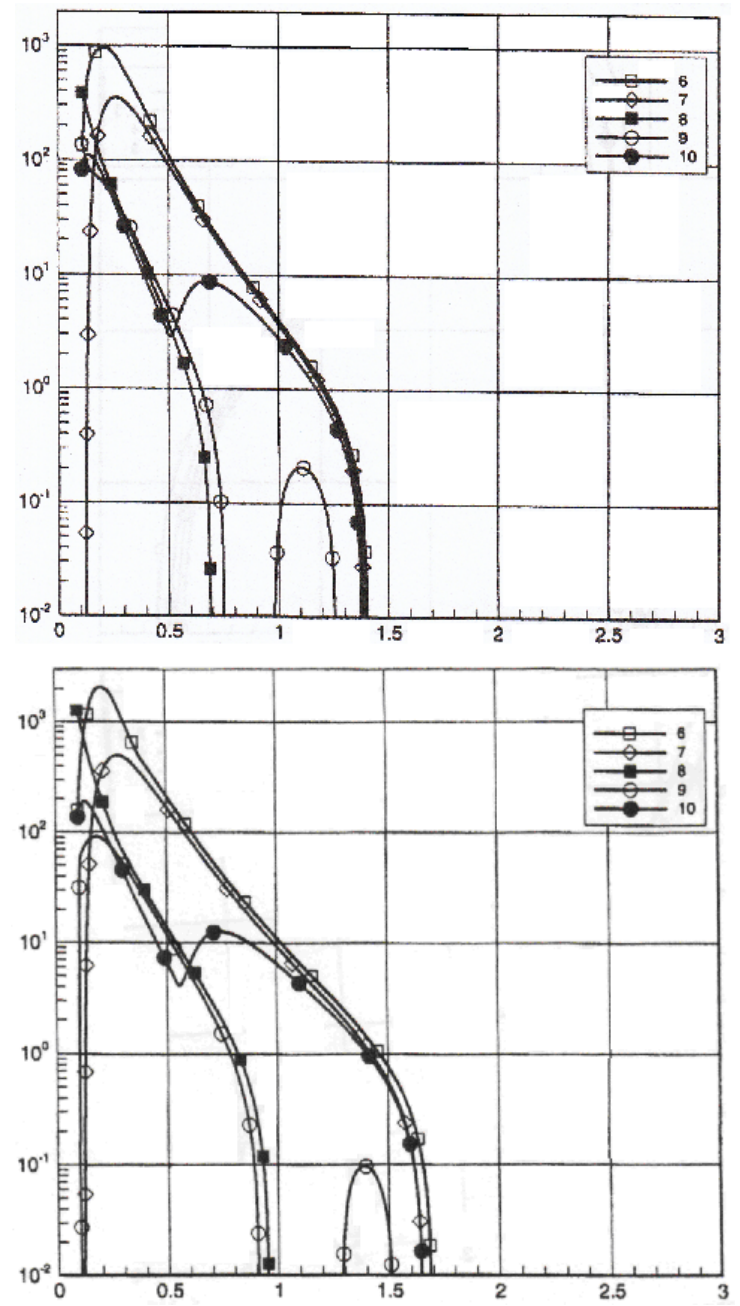

Figure 3: Development of pressure $p\left[\mathrm{~kg} / \mathrm{cm}^{2}\right]$ on the side wall in time [msec]. Upper picture: $q=50 \mathrm{~kg}$, lower picture: $q=100 \mathrm{~kg}$. 


\section{Acknowledgements}

This paper was prepared under the financial support of GAČR, project No. 103/08/0922 and MSM, project number 6840770001.

\section{References}

[1] Lucy, L.B. (1977). A numerical approach to testing of the fission hypothesis. Astron. J. $\quad \mathbf{8 2}, 1013$.

[2] Gingold, R.A. and Monoghan, J.J. (1977). Smooth particle hydrodynamics: theory and application to non-spherical stars. Monthly Nat. R. Astron. Soc. 181, 375.

[3] Randle, P.W. and Libersky, L.D. (1996). Smooth particle hydrodynamics: some recent improvements and application. Appl. Mech. Engng. 139, 175.

[4] Li, S. and Liu, W.K. (2002). Meshfree and particle method and their applications. Appl. Mech. Rev. 55, 1.

[5] Takeda, H., Miyama, S. and Sekiya, M. (1994). Numerical simulation of viscous flow by smoothed particle hydrodynamics. Prog. Theor. Phys. 92, 939.

[6] Chen, J.K., Beraun, J.E. and Carney, T.C. (1999). A corrective smooth particle method for boundary value problems in heat conduction. Int. J. Numer. Methods Engrg. 46, 231.

[7] Veselovsky, A.N., Kurepin, N.S.: Proceedings of $26^{\text {th }}$ Central Scientific Institute of the Russian Federation. Moscow 2006, report II/2, in Russian

[8] Godunov, S.K., Zabrodin, A.V. et.al: Numerical solutions of the polydimensional problem of gas dynamic. Moscow, Nauka, 1976, in Russian.

[9] Zeldovich, J.B., Raize, J.P.: Physics of strike waves and high temperature hydrodynamic processes. Moscow, Nauka, 1966, in Russian.

[10] Prochazka, P., Kravtzov, A. Blast impact on structures of underground parking, Underground spaces, WIT, New Forest 2008. 